\title{
INVESTIGACIÓN
}

Recibido: 28/03/2019 --- Aceptado: 28/05/2019 --- Publicado: 15/09/2020

\section{LA POLÍTICA DEL HASHTAG EN TWITTER}

\author{
Hashtag politics in Twitter
}

Ana Pano Alamán: Università di Bologna. Italia.

ana.pano@unibo.it

\section{RESUMEN}

En este trabajo sobre la comunicación política en Twitter se presentan los resultados de un análisis pragmalingüístico comparado de un corpus de etiquetas o hashtags contenidos en los tuits de cinco líderes políticos españoles, publicados desde el 12 de abril hasta el 8 de noviembre de 2019, en un periodo de casi 8 meses que comprende las elecciones generales, autonómicas y municipales que tuvieron lugar en España ese año y las elecciones al Parlamento europeo. Este estudio cuantitativo y cualitativo tiene como objetivos profundizar en la incidencia que tiene este dispositivo en el discurso político en redes, identificar las semejanzas y diferencias formales y de contenido que presenta en los mensajes de los cinco líderes considerados y señalar sus funciones discursivas. Se pretende así determinar si este es un mecanismo de innovación en la comunicación política actual. Los resultados muestran que existen diferencias en lo que respecta a la cantidad de etiquetas empleada por cada político. Sin embargo, desde un punto de vista formal y funcional, se emplean de manera similar con las funciones de persuadir al votante, como un eslogan, y tematizar y contextualizar el mensaje, invitando implícitamente a los propios seguidores a apoyar y viralizar el contenido político del tuit.

PALABRAS CLAVE: etiqueta - twitter - discurso - enunciación - campaña elecciones

\begin{abstract}
This paper on political communication in Twitter presents and discusses the results of a comparative pragmalinguistic analysis of a corpus of hashtags, included in the tweets published in their own profile by five Spanish political leaders from April 12 to November 8, 2019. Within this period of almost 8 months, two general elections, the elections to the European Parliament, and several regional and local electoral appointments took place in Spain. This quantitative and qualitative study aims at delving into the impact that this device has on political discourse in social network sites, identifying the formal and content similarities and differences that it presents in the messages of the leaders considered, and pointing out the discursive functions that it has in such messages. The main objective is determinining whether hashtags
\end{abstract}


are mechanisms of innovation in current political communication. The results show that there are relevant differences regarding the number of hashtags used by each politician. However, from a formal and a functional point of view, they are used similarly with the functions of persuading the voter, like a slogan, and summing up and contextualizing the content of the tweet. At the same time, the hashtag implicitly invites the leaders' followers to support and viralize the political message.

KEY WORDS: hashtag - twitter - syntax - discourse - enunciation - campaigning elections

\section{A POLITICA DO HASHTAG NO TWITTER}

\section{RESUMO}

Neste trabalho sobre a comunicação política no Twitter se apresentam os resultados de uma análise pragmalingüístico comparado de um corpus de etiquetas ou hashtags contidos nos tuits de cinco líderes políticos espanhóis, publicados desde o 12 de abril até o 8 de novembro de 2019, num período de quase 8 meses que abrange as eleições gerais, autonómicas e municipais que aconteceram na Espanha nesse ano e as eleições ao Parlamento europeu. Este estudo quantitativo e qualitativo tem como objetivos aprofundar a incidência deste dispositivo no discurso político nas redes, identificar as semelhanças e diferenças formais e de conteúdo que apresenta nas mensagens dos cinco líderes considerados e sinalizar suas funções discursivas. É pretendido dessa forma determinar se este é um mecanismo de inovação na comunicação política actual. Os resultados mostram que existem diferenças em relação a quantidade de etiquetas empregadas por cada político. Porém, desde um ponto de vista formal e funcional, se empregam de forma semelhante com as funções de persuadir ao votante, como um slogan, e tematizar e contextualizar a mensagem, convidando implicitamente aos próprios seguidores a apoiar e viralizar o conteúdo político do tuit.

PALAVRAS CHAVE: etiqueta - twitter - discurso - enunciação - campanha eleiçoes

\section{Como citar el artículo:}

Pano Alamán, A. (2020). La política del hashtag en Twitter. [Hashtag politics in Twitter]. Vivat Academia. Revista de Comunicación, 152, 49-68. doi: http:/ / doi.org/10.15178/va.2020.152.49-68 Recuperado de:

http://www.vivatacademia.net/index.php/vivat/article/view/1240

\section{INTRODUCCIÓN}

La etiqueta o hashtag es uno de los dispositivos más utilizados por los usuarios de Twitter, no obstante, debido a su alcance comunicativo y gran versatilidad, ha 
traspasado las fronteras del microblog, siendo muy habitual ver el signo \# que la caracteriza en pancartas, publicidad televisiva o fotografías (Heid y Puschmann, 2017). Los hashtags, que surgieron en 2009 para etiquetar contenido en Twitter, son cadenas de caracteres precedidas del símbolo \# que se convierten automáticamente en enlaces y que pueden rastrearse en el buscador del microblog. Generalmente, se trata de palabras o secuencias de pocas palabras que permiten marcar el tema de un tuit y decir más con menos, esto es, concentrando en esas pocas palabras distintas funciones informativas, persuasivas, argumentativas o expresivas y promoviendo la interacción entre usuarios (Mancera Rueda y Pano Alamán, 2015). En el ámbito de la política, se han convertido en un dispositivo que, de manera similar a los emojis y a los vídeos incrustados en el texto, han contribuido a transformar el discurso político en clave multimodal e hipertextual (Pano Alamán, 2019).

El objetivo de este estudio es contribuir a la investigación sobre el papel que tiene este dispositivo en las interacciones que se producen en Twitter y determinar en qué medida contribuye a innovar las estrategias de comunicación política, teniendo en cuenta las mutaciones que se producen hoy en discurso de los políticos en las redes (Gallardo Paúls y Enguix Oliver, 2016; Pellisser Rossell y Oleaque Moreno, 2019). En concreto, se presentan los resultados de un análisis lingüísticopragmático comparado del uso que hacen de las etiquetas cinco líderes políticos españoles entre el 12 de abril y el 8 de noviembre de 2019. El estudio busca dar respuesta a las preguntas: ¿qué etiquetas emplearon los políticos españoles en Twitter durante ese periodo?, ¿qué rasgos lingüísticos presentan en los tuits analizados?, y ¿qué funciones discursivas desempeñan? Tras revisar distintos aspectos teóricos relacionados con las etiquetas en la comunicación política y presentar la metodología y el corpus de estudio, se presentan y discuten los resultados, aportando algunas reflexiones sobre la investigación en este ámbito.

\section{MARCO TEÓRICO}

El hashtag o etiqueta se comporta en Twitter como metadato explícito integrado en el tuit, indicando cuál es el tema del mismo y asociándolo a otros tuits publicados en distintos perfiles, en los que se integra esa misma etiqueta. En este sentido, sitúa el microblog en el mundo de la folksonomía, pues constituye también un método de clasificación colaborativa de datos a partir de palabras clave elegidas por los usuarios de la red. De este modo, la etiqueta amplía el significado potencial del tuit, puesto que, convirtiéndose en un tag que es posible enlazar y buscar, se relaciona con otros discursos, promoviendo lo que Zappavigna llama "ambiente de afiliación" (2011). Si se inserta la etiqueta en el buscador de Twitter, es posible recuperar un tuit o un conjunto de tuits relacionados con el tema que indica y seguir la "conversación" que se desarrolla sobre el mismo. En definitiva, gracias a este dispositivo, los usuarios pueden recapitular ideas e incluso acceder a distintos hilos e interacciones mediante el uso de esa "palabra clave".

Desde el análisis del discurso, el hashtag se ha analizado como dispositivo que promueve el ciberactivismo en las redes (Menna, 2012; Zeifer, 2020), el debate y la 
participación política de los ciudadanos en torno a una misma temática (Santoveña, 2015; Escuder, 2019), la configuración de la propia marca e imagen (Page, 2012), como mecanismo que permite analizar el debate que se produce, por ejemplo, en un programa de televisión, como si Twitter fuera una "segunda pantalla" (Calvo Rubio, 2018), como elemento de creación de la agenda mediática (Briceño Romero et al., 2018) o de encuadre de noticias (Pérez Fumero, 2013). En estas investigaciones, se asume que el signo \# es un elemento semiótico complejo que establece distintos tipos de relaciones con el resto de los constituyentes del tuit. Menna (2012, p. 49) apunta a tres tipos de relaciones: interna, referida a la vinculación de la etiqueta con los elementos lingüísticos del mensaje; externa, que alude a las relaciones posibles entre el contenido del mensaje en que aparece la etiqueta y el contenido de los mensajes que también la utilizan (por ejemplo, cuando se transforma en hipervínculo para acceder a todos los tuits que la contienen); y contextual, que es la relación que se establece entre una unidad significante, en este caso un tuit, y el contexto situacional al cual remite, generando significados que el destinatario interpreta a partir de los conocimientos que comparte con el emisor (Heyd y Puschmann, 2017, p. 56). En este sentido, es importante señalar que:

Hashtags provide a means by which tweeters can activate relevant contextual assumptions within the character limit and without the need to provide explicit background information and thus detract from the casual, informal style. In sum, hashtags can be used to activate certain contextual assumptions, thus guiding the reader's inferential processes (Scott, 2015, p. 19).

Dentro del análisis del discurso político español, se ha señalado que los tuits vehiculan un discurso sintético y fragmentado (Mancera Rueda y Pano Alamán, 2013; Pano Alamán y Mancera Rueda, 2014). El discurso político en Twitter se conecta, de hecho, con un discurso político actual en el que la información se apoya en pocas y contundentes palabras, con las que cabe "decir poco, repetirlo mucho y, a ser posible, nada o poco explicar" (Ortega, 2005, p. 23) y que, de acuerdo con Gallardo-Paúls y Enguix Oliver (2016), se desplaza hacia un discurso pseudopolítico, caracterizado por el personalismo narcisista en el mensaje de los representantes, la espectacularización frivolizante en los medios de comunicación y una desideologización en el discurso de los ciudadanos. Por otra parte, el mensaje político en Twitter se caracteriza, de un lado, por la fragmentación, puesto que presenta la información en porciones -texto, emojis, imágenes, enlaces, etiquetas, vídeos, fotografías y carteles- que interrumpen el flujo comunicativo; y, de otro lado, por la condensación semántica (Held, 2011, pp. 35-36), en la medida en que el enunciado se apoya generalmente en estructuras elípticas y en pocas palabras plenas (sustantivos, verbos, adjetivos, adverbios) (Pano Alamán, 2019). Quizá el elemento que mejor expresa la tendencia del tuit político a la fragmentación y la condensación es el hashtag. En relación con sus funciones en el discurso político, Mancera Rueda y Helfrich (2014) han señalado que las etiquetas permiten clasificar los tuits en mensajes de apoyo hacia el gobierno y mensajes de crítica al gobierno por parte de los partidos en la oposición. Asimismo, mediante las etiquetas, los políticos elaboran una "retórica de la persuasión" destinada a sus seguidores en el microblog o, en 
general, a sus simpatizantes, para mostrar que están “en primera línea del debate público" (Mancera Rueda y Helfrich, 2014, p. 83).

\subsection{Del eslogan a la etiqueta en campaña}

Cabe señalar que las etiquetas en los tuits de los políticos aparecen a menudo al final del mensaje, a veces sin integración sintáctica con el resto de elementos verbales y no verbales, de manera que se visualizan rápidamente resumiendo el contenido del tuit (Mancera Rueda y Pano Alamán, 2015). En este sentido, pueden funcionar a modo de consigna o eslogan, puesto que buscan obtener una reacción en el destinatario que lo lleve a identificarse o a respaldar lo que plantea el emisor político, especialmente en periodos de campaña electoral (Mancera Rueda y Pano Alamán, 2013). Por ejemplo, en la primera campaña de las elecciones generales españolas desarrollada en Twitter, el equipo del candidato Alfredo Pérez Rubalcaba lanzó -a raíz de la celebración del debate cara a cara entre este y Mariano Rajoy el 7 de noviembre de 2011- la etiqueta \#undebatedecisivo, para que quien seguía el perfil de este político pudiese expresar su opinión sobre dicho encuentro, insertando dicha etiqueta es sus tuits. La búsqueda en Twitter de la secuencia \#undebatedecisivo arrojaba numerosos mensajes tanto positivos como negativos sobre el cara a cara, por lo que su empleo por parte de los usuarios en Twitter dio resultados inesperados, tanto favorables como adversos para la estrategia del partido socialista. El hashtag nació también con la pretensión de convertirse en uno de los Trending Topic ${ }^{1}$ más comentados en España. Sin embargo, aunque efectivamente acabó convirtiéndose en TT, también provocó una oleada de opiniones críticas que denotaban una indiferencia general de los internautas hacia el debate o que ponían en tela de juicio precisamente el carácter decisivo del encuentro. En otros casos, la misma etiqueta se empleó, como había sido previsto, para señalar y agrupar aquellos mensajes que podían contener propuestas útiles para el debate. El análisis de las etiquetas utilizadas durante la campaña de las elecciones al Parlamento europeo de 2014 (Mancera Rueda y Pano Alamán 2015) y sobre las más frecuentes de la campaña de las generales de 2015 (González Bengoechea, 2015) confirman este mismo empleo de los hashtags. En efecto, como el eslogan, la etiqueta parece designar "una fórmula concisa, fácil de retener y de repetir, que implica o connota y sugiere más que lo que realmente dice o denota" (López Eire (1998, p. 43). Asimismo, se caracteriza por su brevedad; es, en definitiva, una unidad textual autónoma que, en el discurso político en las redes, sintetiza el mensaje anclándolo al partido o al candidato.

Tanto el eslogan como la etiqueta presentan un alto grado de densidad semántica, que se manifiesta en el uso de palabras plenas como sustantivos, verbos, adjetivos y adverbios que vehiculan un mensaje sencillo, para alcanzar un público lo más amplio

\footnotetext{
${ }^{1}$ El tema indicado por la etiqueta puede convertirse en un TT cuando el número de mensajes que la contienen aumenta en un período de tiempo breve. Generalmente se trata del término o del sintagma más comentado por los usuarios en un corto lapso de tiempo. El sistema rastrea automáticamente esos términos y, por medio de un cómputo de la frecuencia de aparición de la palabra o del sintagma, determina qué etiqueta sobresale por encima de las demás.
} 
posible (Peña Jiménez y Ortiz Sobrino, 2011, p. 553). Por otra parte, el eslogan, como la etiqueta empleada con fines electorales, serán más evocadores si son fáciles de percibir, interpretar y memorizar (Reboul, 1978, p. 42). No en vano, como en el caso del eslogan publicitario, la brevedad y densidad del eslogan político responden a su vocación persuasiva (Garrido-Lora, 2013, p. 177). Otra de las características del eslogan, al que aspiran algunos hashtags, es la brillantez, que contribuye a que sea recordado y que se apoya, en parte, en el contenido semántico de las palabras y en los recursos formales que se ponen en juego para elaborarlo. Como recuerda Garrido Lora (2013, pp. 178-179), los eslóganes utilizados en la campaña electoral de las generales de 2008 incorporaban figuras retóricas como el paralelismo, la hipérbole o la elipsis y recursos tipográficos como las mayúsculas, para dar expresividad.

\section{METODOLOGÍA y CORPUS}

Con el objeto de determinar cómo se comportan discursivamente las etiquetas en los mensajes en Twitter de los principales líderes de cinco partidos políticos españoles, se ha llevado a cabo un análisis cuantitativo-cualitativo y comparado de un corpus de tuits publicados en sus perfiles. En primer lugar, con Sketch Engine se han obtenido una lista de frecuencias de los hashtag más frecuentes y las concordancias relativas a cada uno. En segundo lugar, se han identificado sus funciones en los tuits siguiendo la propuesta de Mancera Rueda y Pano Alamán (2015) para el análisis cualitativo de los valores sintáctico-discursivos de las etiquetas en estos discursos políticos. Para constituir el corpus, primero se han recopilado automáticamente (mediante la API de Twitter) los tuits publicados en los perfiles oficiales de Pedro Sánchez (PSOE), Pablo Casado (PP), Pablo Iglesias (Unidas Podemos), Albert Rivera (Ciudadanos) y Santiago Abascal (Vox), entre el 12 de abril de 2019, que marca el inicio de la campaña electoral del 28A, fecha de las elecciones generales y de las autonómicas en la Comunidad Valenciana, hasta el 8 de noviembre de 2019, día de cierre de la campaña del 10N. Entre esas dos convocatorias tuvieron lugar también, el 26 de mayo (26M), las elecciones al Parlamento europeo, las autonómicas, a excepción de las consideradas históricas y de la Comunidad Valenciana, y las municipales en todo el territorio. El número total de tuits descargados es 3905, excluyendo los retuits o reenvíos de mensajes de otros usuarios. La Tabla 1 indica el total de tuits recogidos, el número total de etiquetas empleadas y el número de etiquetas únicas, distribuidos por perfil:

Tabla 1. Número de tuits recopilados y corpus de etiquetas por perfil.

\begin{tabular}{|l|l|l|l|}
\hline Perfiles & Tuits & N. hashtags totales & N. hashtags únicos \\
\hline @sanchezcastejon & 966 & 1481 & 434 \\
\hline @pablocasado__ & 867 & 301 & 126 \\
\hline @PabloIglesias & 700 & 175 & 103 \\
\hline @Albert_Rivera & 706 & 433 & 164 \\
\hline @Santi_ABASCAL & 458 & 352 & 120 \\
\hline TOTAL & $\mathbf{3 9 0 5}$ & $\mathbf{3 1 0 9}$ & $\mathbf{9 6 9}$ \\
\hline
\end{tabular}

Fuente: Elaboración propia. 
Pano Alamán, A.

La política del hashtag en Twitter

\section{RESULTADOS}

\subsection{Análisis cuantitativo}

En el total de tuits recogidos se han empleado 3109 etiquetas totales y 969 etiquetas únicas o distintas (v. Tabla 1). Esta diferencia es relevante, puesto que no existe una correspondencia exacta entre el número de tuits publicados y número de etiquetas empleadas: por una parte, no todos los mensajes contiene hashtags, por otra, es frecuente que en un mismo texto aparezcan varias etiquetas. Desde el punto de vista de la frecuencia, notamos algunas diferencias que apuntan a distintas estrategias de uso de este dispositivo por parte de los políticos.

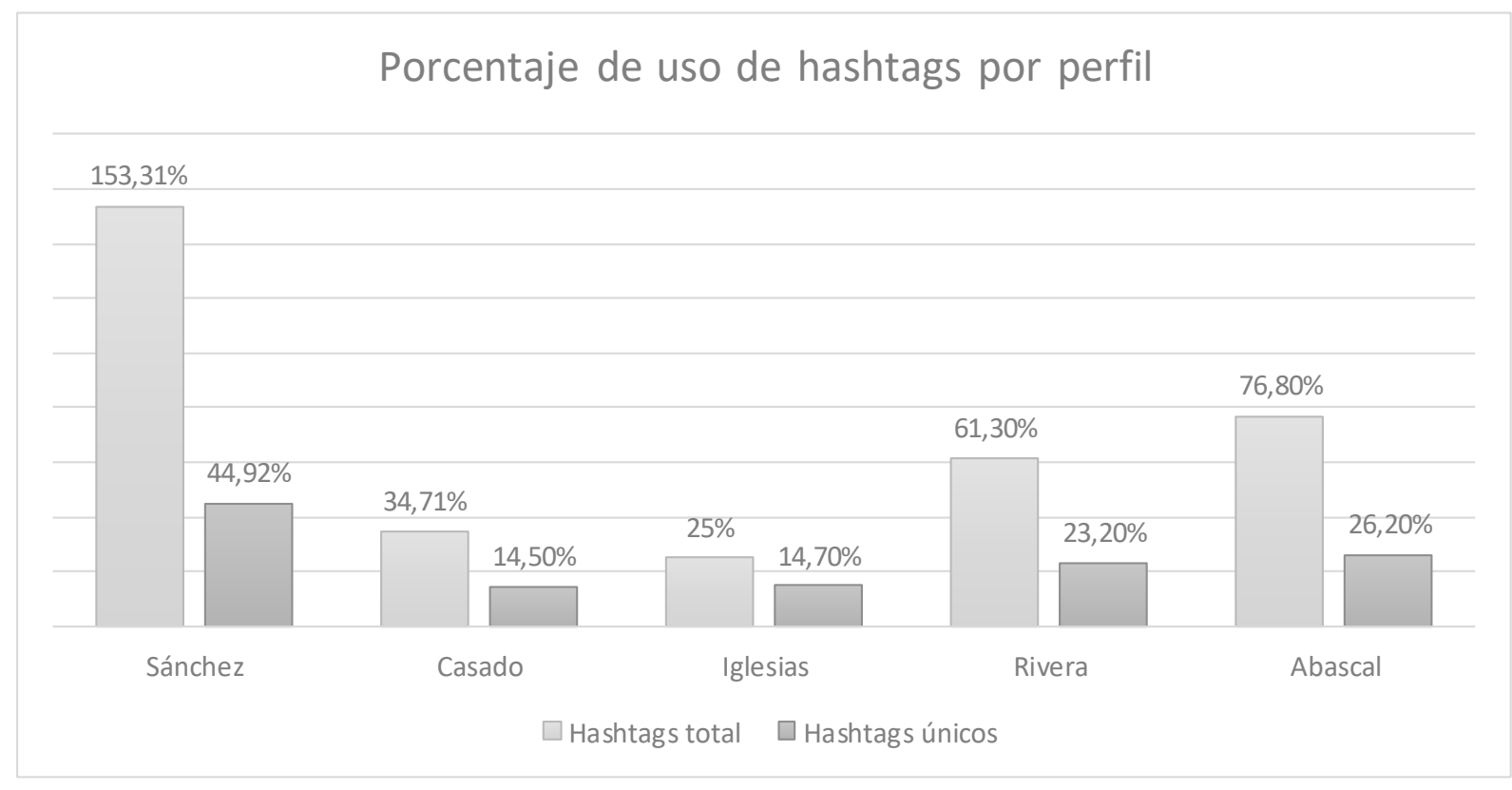

Gráfico 1: Porcentaje ponderado de hashtags totales y únicos (no repetidos) por perfil. Fuente: Elaboración propia.

Como puede notarse, el porcentaje de uso total de Sánchez $(153,31 \%)$ supera el de los demás e indica que el político socialista empleó en general más de una etiqueta en sus mensajes. Le siguen Abascal (76,8\%) y Rivera (61,30\%) y, más distanciados, se hallan Casado (34,71\%) e Iglesias (25\%). En cuanto al porcentaje de etiquetas únicas, observamos que las diferencias entre políticos se acortan. En este caso, cabe señalar que el líder del PSOE, si bien emplea numerosos hashtags, estos suelen repetirse a menudo dado que menos de la mitad del total (44,92\%) son etiquetas distintas. Algo similar se observa en Abascal $(26,20 \%)$ y Rivera $(23,20 \%)$, con un porcentaje similar de etiquetas únicas. En los casos de Casado e Iglesias, la distancia entre porcentaje total y único es menor. Por otra parte, el líder de Podemos es quien menos utilizó este dispositivo, aunque lo hizo de manera más diversificada respecto a los demás. 
Sánchez es, pues, quien emplea un mayor número de etiquetas totales y únicas, un dato que confirma la tendencia observada en el estudio de la campaña electoral de 2011, en la que el entonces candidato socialista, Pérez Rubalcaba, utilizó un total de 105 etiquetas frente a las 43 empleadas por Mariano Rajoy (Mancera Rueda y Pano Alamán, 2013, p. 193 Los resultados del análisis de González Bengoechea (2015, p. 549) sobre el uso de Twitter por distintos partidos españoles entre el 23 y el 29 de abril de 2015, muestran que PSOE y Podemos fueron los partidos que más utilizaron este dispositivo. Mientras Vox, entonces "partido emergente", daba poco espacio a este recurso con apenas 4 etiquetas, Podemos lo utilizó en 135 ocasiones, llegando a emplear hasta en 66 veces el hashtag \#L6Ncalleiglesias, para informar de lo que decía su líder en el programa La Sexta Noche de La Sexta. Más recientemente, el análisis de una muestra de tuits publicados entre el 1 de julio y el 17 de agosto de 2018, en un periodo inactivo desde un punto de vista electoral e institucional (Pano Alamán, 2019), indican que Podemos fue el partido que menos utilizó el hashtag (29,2\%) frente al PSOE, que lo empleó en el $87 \%$ de tuits publicados, Ciudadanos (74\%) y PP $(43,8 \%)$. Estos últimos datos coinciden en parte con los resultados de esta investigación, lo cual lleva a pensar que el mayor o menor uso de etiquetas no depende tanto del periodo en que se publican los mensajes, esto es, en campaña preelectoral, electoral o fuera de campaña, cuanto de la estrategia comunicativa del partido y de su líder en Twitter. Asimismo, cabe señalar la contratendencia en el caso de UnidasPodemos y Vox respecto a campañas precedentes. Mientras Iglesias reduce el número de hastags empleados, Abascal incrementa su empleo, debido en parte a la urgencia de las citas electorales en 2019 y al peso que tiene hoy en día en el sistema representativo español.

En cuanto a las etiquetas más frecuentes en el corpus, la Tabla 2 recoge las que han sido empleadas al menos en 5 ocasiones por cada uno de los políticos considerados:

Tabla 2. Etiquetas más frecuentes por perfil.

\begin{tabular}{|l|l|}
\hline Perfiles & Etiquetas más frecuentes > 5 \\
\hline @sanchezcastejon & \#HazQuePase (50), \#10N (61), \#AhoraSí (58), \#SiempreHaciaDelante (56), \#26M \\
& (55), \#AhoraEspaña (48), \#28A (41), \#EstamosMuyCerca (37), \#AhoraUnidad \\
& (30), \#España (25), \#ConstruirGobierno (23), \#ViolenciaMachista (22). \#EUCO \\
& (13), \#ViolenciaDeGénero (13), \#NiUnaMenos (12), \#LaEspañaQueQuieres (12), \\
& \#VotaConElCorazón/ O (9), \#ProgramaComúnProgresista (8), \#SánchezARV \\
& (8), \#G20OsakaSummit (8), \#ElDebate4N (8), \#Agenda2030 (8), \\
& \#TransiciónEcológica (7), \#CMin (7), \#Gobierno (7), \#Europa (6), \#G20 (6), \\
& \#igualdad (5), \#AhoraAvanzar (5), \#AhoraPrograma (5), \#DANA (5), \\
& \#ElDebateDecisivo (5), \#G20Osaka (5), \#LaEuropaQueQuieres (5), \\
& \#PlanAntibloqueo (5), \#SánchezA3N (5), \#SelFEM (5), \#SesiónDeInvestidura (5) \\
\hline @pablocasado__ & \#ValorSeguro (32), \#VotaPP (26), \#26M (21), \#10N (16), \#28A (11), \\
& \#CentradosEnTuFuturo (11), \#NosUneEspaña (9), \#ElVotoSeUne (8), \\
& \#PorTodoLoQueNosUne (7), \#CasadoEnOndaCero (5), \#ElDebateDecisivo (5), \\
& \#ELDEBATEenRTVE (5) \\
\hline @PabloIglesias & \#LaHistoriaLaEscribesTú (19), \#UnGobiernoContigo (9), \#SíSePuede (5) \\
\hline @Albert_Rivera & \#NoNosCallarán (18), \#EspañaEnMarcha (17), \#VamosCiudadanos (12), \#28A \\
& (9), \#26M (7), \#VamosPorLaLibertad (6) \\
\hline
\end{tabular}




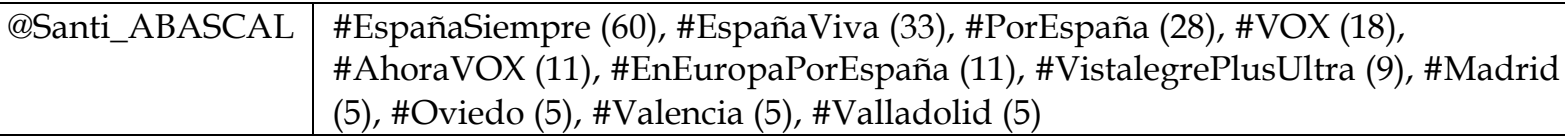

Fuente: Sketch Engine y elaboración propia.

Como se puede observar, algunas de las etiquetas más empleadas aparecen en más de un perfil. Véase, por ejemplo, el caso de \#28A y \#26M, que aluden a las fechas de las primeras elecciones generales de 2019 y de las europeas, y que aparecen en los mensajes de Sánchez, Casado y Rivera $61(28 \mathrm{~A}), 77(10 \mathrm{~N})$ y 83 veces $(26 \mathrm{M})$. En todo caso, las etiquetas más frecuentes son sintagmas verbales y nominales que funcionan como consignas y lemas, un dato que no sorprende dada la concentración en 2019 de diversas citas electorales. Todos los perfiles analizados utilizan etiquetas que coinciden con los lemas oficiales adoptados por los partidos en la campaña del 28A o el 10N, como: \#HazQuePase, utilizada 50 veces por Sánchez; \#EspañaSiempre, que le sigue con 60 ocurrencias en los tuits de Abascal; \#ValorSeguro, que constituye la principal en los de Casado con 32 ocurrencias; \#LaHistoriaLaEscribesTú, que aparece en 19 ocasiones en los mensajes de Iglesias; y \#EspañaEnMarcha, con 17 ocurrencias en los de Rivera. Es curioso que, solo en este último caso, este hashtag no sea el que aparece más veces, pues en los tuits del entonces líder de Ciudadanos el más utilizado fue el sintagma verbal en forma negativa: \#NoNosCallarán, presente en 18 ocasiones. Otras etiquetas frecuentes en el corpus son los eslóganes utilizados en los periodos de precampaña y las consignas que se acumulan en los mensajes de final de campaña para exhortar a los electores a votar a uno u otro partido o recordar el motivo por el que hay que votarles: \#LaEspañaQueQuieres, \#VotaPP, \#SíSePuede, \#VamosPorLaLibertad o \#EspañaViva.

Más allá de este tipo de etiquetas electorales, son frecuentes también aquellas que, solo en el caso del líder socialista, aluden a temáticas relacionadas con las políticas del Gobierno entonces presidido por Sánchez, o de su partido, y temas relacionados con la actualidad que se debatían en la opinión pública. En particular, destacan \#ViolenciaMachista, \#ViolenciaDeGénero y \#NiUnaMenos, con 21, 13 y 12 ocurrencias cada una, que ponen la atención sobre los feminicidios; otras etiquetas temáticas frecuentes en los tuits de Sánchez son las relacionadas con las políticas de gobierno, anunciadas a través de etiquetas como \#Agenda2030, \#TransiciónEcológica o \#igualdad. Son muy habituales también en su perfil los acrónimos y abreviaturas que hacen referencia a momentos y lugares específicos, como las cumbres europeas e internacionales: \#EUCO (Consejo Europeo), que aparece en 13 ocasiones, y \#G20OsakaSummit, \#G20 y \#G20Osaka, relativas a la cumbre de los países del G-20 que tuvo lugar el 28-29 de junio de 2019, que suman en total 19 ocurrencias. Son espacios en los que Sánchez participa en ese momento como presidente del gobierno y que por este motivo no se encuentran en otros perfiles o presentan una frecuencia mucho menor.

Este tipo de etiqueta, que denominamos contextualizadora, aparece también en los mensajes de los otros políticos considerados. Ya hemos visto las fechas que indican el 
día de elecciones, a las que se unen aquellas etiquetas que indican un topónimo en el que tiene lugar un mítin electoral, como son \#Madrid, empleada en 5 ocasiones por Sánchez y Abascal, en cuyos mensajes se repiten también hasta cinco veces \#Oviedo, \#Valencia y \#Valladolid. Esto permite afirmar que Abascal utiliza este dispositivo prevalentemente para dar un contexto a sus palabras y acciones, sobre todo en campaña, además de dar un guiño a esas ciudades y a sus residentes para ganarse a sus potenciales electores. Por último, merece la pena detenerse en los hashtags que unen en un mismo sintagma el nombre del líder y el nombre de un programa de televisión o radio en el que este está siendo entrevistado. Se trata de etiquetas que aparecen en todos los perfiles, aunque con una presencia mayor en los tuits del líder socialista (\#SánchezARV, SánchezA3N) y en los de Casado (\#CasadoEnOndaCero). De manera similar se emplean las etiquetas \#ElDebate4N y \#ElDebateDecisivo, que hacen referencia a los debates cara a cara que tuvieron lugar durante las campañas.

\subsection{Análisis cualitativo}

En relación con los rasgos lingüísticos más destacados cabe señalar que la mayor parte de los hashtags utilizados por los líderes políticos son palabras, generalmente sustantivos, pertenecientes al léxico general o especializado, que aluden a temas que en esos momentos son de actualidad. Este tipo de etiquetas son más frecuentes en el perfil de Sánchez, donde se combinan sustantivos relacionados con las políticas del gobierno y con las propuestas y promesas del partido de cara a las elecciones (\#agua, \#cultura, \#digitalización, \#emprendimiento, \#pobreza, \#startup, \#tuberculosis). Estas son, en cambio, más raras en los mensajes Abascal, con solo cinco ocurrencias (\#agricultores, \#autonómicas, \#congreso, \#Lema, \#sentencia) y en los de Rivera, con cuatro (\#gotafría, \#paro, \#selectividad, \#Trial). Son prácticamente anecdóticas con un único ejemplo en Casado (\#HepatitisC) e Iglesias (\#Selectividad). Este tipo de hashtag se inserta en la estructura sintáctica del enunciado en el que desempeña una función. Por ejemplo, en (1), \#convivencia es objeto directo del verbo "querer", que designa lo que quiere la sociedad catalana según Sánchez, y en (2), \#paro es sujeto en un enunciado asertivo de Rivera en el que cita los datos de desocupación publicados en septiembre de 2019 para atacar las políticas económicas y fiscales del Gobierno:

(1) @sanchezcastejon: Siempre hemos defendido la ley y el diálogo en Cataluña, pero el independentismo sigue tropezando con la misma piedra. La sociedad catalana no quiere independizarse, lo decide en cada proceso electoral. Quiere \#convivencia y eso es lo que debe garantizar la Generalitat (0310-2019).

(2) @Albert_Rivera: El \#paro registra el peor dato en un mes de agosto desde 2010 y España destruye 213.000 empleos. Disparar el gasto, subir impuestos a familias y emprendedores, aparcar las reformas pendientes y podemizar la economía tiene consecuencias (03-09-2019).

Otro tipo de etiquetas con un único elemento son aquellas que aluden, como decíamos, a ciudades o comunidades autónomas que en el mensaje hacen referencia al lugar en el que se encuentran los políticos, en particular, en los mítines electorales. 
Pano Alamán, A.

La política del hashtag en Twitter

En los mensajes de Sánchez aparecen 65 ocurrencias únicas de ciudades y CCAA, en los de Abascal 47, datos que contrastan con los de Casado (4) e Iglesias, que emplea solo una etiqueta de este tipo, \#Murcia, con función tematizadora, en un tuit que denuncia la contaminación por parte de multinacionales en esa región. En todo caso, las etiquetas más frecuentes son las que se apoyan en sintagmas nominales más complejos, en las que las lexías aparecen fusionadas. Generalmente, estas incluyen sustantivos precedidos o seguidos de adjetivos:

(3) @PabloIglesias: Hay quienes tienen mucho poder en las instituciones europeas sin pasar por las urnas. Unidas Podemos Cambiar Europa [...] trabajará por una Europa más democrática, que sirva a la gente y no a los lobbies. \#UnaEuropaMásJusta (14-05-2019).

(4) @Santi_ABASCAL: Terrible vídeo: El número 3 del PP mintiendo sobre VOX y, de rodillas, pidiendo perdón a BILDU. Merecen desaparecer en las urnas \#DerechitaCobarde (12-04-2019)

También son habituales los sustantivos seguidos de otros sustantivos y siglas en aposición, como en los siguientes tuits:

(5) @sanchezcastejon: Gracias por vuestro compromiso municipalista. Estoy seguro de que haréis un gran trabajo trasladando los valores socialistas a las políticas públicas de vuestros pueblos y ciudades para mejorar la vida de la gente. ¡Felicidades, compañeros y compañeras! \#AytosPSOE (15-06-2019).

(6) @pablocasado_: Clara, concisa y coherente, @cayetanaAT ha demostrado ser la mejor candidata del \#DebateRTVE. [...] Enhorabuena (16-04-2019).

Por otro lado, abundan en todos los perfiles las etiquetas en las que el sustantivo aparece seguido de complemento de nombre como en \#CopaDelRey (Rivera) y \#EstadoDeExcepción (Abascal). También se nota una abundante presencia en todos los perfiles y sin distinción de hashtags que aluden, por un lado, a instituciones como el Congreso (\#congreso, \#congresodelosdiputados), el Consejo de Ministros (\#CMin, en el caso de Sánchez) o Consejo Europeo (\#EUCO) y, por otro, a celebraciones. La fórmula más habitual en estos casos es la de \#Díade+ComunidadAutónoma que, si bien aparece en todos los perfiles, abunda en particular en los de Sánchez (29) y Rivera (19); también se repiten en más de un perfil otras como \#DíaDasLetrasGalegas (Sánchez, Iglesias) o \#DíaDeEuropa (Sánchez, Casado, Rivera, Iglesias). A menudo, Sánchez, Casado y Rivera acuden a este dispositivo para desear un \#FelizJueves, \#FelizDomingo o incluso el más coloquial \#FelizFinde. En ocasiones, la celebración se relaciona con "el día mundial" de los derechos de determinados grupos sociales o bien con onomásticas. En este sentido, las diferencias son notables, pues cada político selecciona celebraciones distintas en función de su ideología y de la que supone en sus seguidores en Twitter, para elaborar mensajes en los que se reivindican su posición ante determinadas cuestiones sociales y políticas que marcan su programa:

(7) @PabloIglesias: 25 personas por minuto son obligadas a abandonar su hogar por causa de las guerras, el hambre y la persecución. Uno de cada dos 
refugiados son niños o niñas. Hoy es el \#DiaMundialDelRefugiado. Todos los días, mirar hacia otro lado nos vuelve indiferentes ante la barbarie (20-062019).

(8) @Santi_ABASCAL: Ha sido muy emocionante disfrutar del desfile de las Fuerzas Armadas en el Día de la Hispanidad. La entrega de tantos hombres y mujeres a la Patria y al bien común nos da esperanza a todos los españoles. \#DíaDeLaHispanidad \#DíaDelPilar \#12oct (12-10-2019).

(9) @Albert_Rivera: Los catalanes constitucionalistas no podemos celebrar la fiesta de nuestra tierra porque los separatistas la han convertido en la fiesta del odio a España. Pero sí puede celebrarla Otegi, que todavía no ha pedido perdón por las matanzas de su banda en Hipercor o Vic. \#Diada2019 (11-09-2019).

Por último, se puede señalar también la presencia notable en todos los perfiles, menos en el de Abascal, de etiquetas con siglas y acrónimos que hacen referencia, en la mayor parte de los casos, a equipos y eventos deportivos (\#FIFAWWC, \#Sub21, \#ATPMontreal, \#SelFEM, \#NBA, \#RG29, \#MX2)2 ${ }^{2}$, especialmente en los mensajes de Rivera, aparentemente más atento a estos acontecimientos. Otras señalan cuestiones que ocupan la agenda mediática, como \#IIFF (incendios forestales), etiqueta que emplean Sánchez, Casado y Rivera únicamente, en esta forma o con la estructura \#IF+Lugar, o bien propuestas políticas, como \#PPR (Prisión Permanente Revisable) en los mensajes de Casado, y efemérides, como \#DIFAS2019 (Día de las Fuerzas Armadas 2019), que aparece en un mensaje de Rivera. En relación con las siglas, es posible afirmar que los partidos privilegian aquellas que señalan acontecimientos deportivos nacionales e internacionales, alejados de las propuestas políticas que contienen sus programas o de los temas que caracterizan sus propuestas políticas, confirmando la tendencia ya observada de los contenidos desideologizados que vehiculan en las redes (Gallardo Paúls y Enguix Oliver, 2016).

En todo caso y, a pesar de que los sintagmas nominales son predominantes, se emplean en algunos casos sintagmas verbales, tanto en estructuras copulativas como \#155EsLibertad (Rivera), \#EsTiempoDeMemoria (Sánchez) y \#SoyEspañol (Abascal), o en la etiqueta con significado deportivo \#SomosEquipo (Sánchez, Casado y Rivera), como en estructuras predicativas, cuyo significado es más complejo y requiere un esfuerzo de interpretación mayor en base al contexto:

(10) @Albert_Rivera: Mis compromisos con la infancia, el futuro de España: Ley de erradicación de la violencia contra la infanciaQue no prescriban los delitos de abusos sexuales a niños Plan de ayudas a todas las familias Gracias, @unicef_es. \#AunqueNoVoten (22-04-2019).

(11) @pablocasado_: Bildu desvela que son los interlocutores prioritarios del PSOE, con quien se ha comprometido a mantener una colaboración continua.

\footnotetext{
2 Véanse también \#SOSMarMenor (Sánchez, Iglesias), \#SOSPrisiones21M (Rivera), \#StopOkupas (Casado) y \#STOPDelincuencia (Rivera).
} 
Es insólito que exija nuestra abstención quien negocia con los defensores de ETA.

\#SánchezNavarraNoSeVende (27-07-2019).

Otras etiquetas contienen verbos en presente de indicativo con los que los políticos buscan posicionarse ante hechos de actualidad (\#yosítecreo, Iglesias), denunciar o llamar la atención sobre algo que está ocurriendo (\#BarcelonaArde, Abascal), lanzar ideas o soluciones ante determinados problemas (\#RompeElCírculo, Sánchez) o vehicular consignas y lemas electorales (\#NosUneElEmpleo, Casado). En los tuits de Sánchez, por otra parte, aparecen también etiquetas con verbos en infinitivo, como \#AhoraAvanzar o \#ConstruirDeporte, mientras que destaca en los de Iglesias y Abascal el recurso a estructuras modales como la irónica \#aversivoyaserdeUP del primero y \#NoQueremosVerAOtegi del segundo. En el caso de Rivera cabe señalar el empleo repetido de la expresión "vamos" en \#VamosJuntos, \#VamosMoteros o incluso en la perífrasis \#VamosAGanar, que funciona también como lema de la campaña del 28A. Por último, en los mensajes de todos los líderes hay etiquetas en las que se incluye el imperativo en enunciados exhortativos que piden al elector su voto, coincidiendo en parte con el lema electoral: \#HazQuePase, \#VotaPP, \#VotaVox, \#VamosPorLaLibertad, \#ApodérateUnidasPodemos.

En las etiquetas de Casado y Rivera es más frecuente el nosotros que incluye tanto al político como al potencial votante, véanse: \#ElVotoQueNosUne (Casado) y \#VamosCiudadanos (Rivera). En los mensajes de Iglesias y Abascal, ese significado inclusivo pasa, en cambio, a través del yo con el que el cada líder político invita a sus seguidores a identificarse: \#YoRepresentoaPodemos (Iglesias) o \#YoVoyaVistalegre (Abascal). El pronombre personal tú aparece explícito solo en los hashtags de Iglesias (\#LaHistoriaLaEscribesTú, \#LaRegiónLaHacesTú), mientras que en el resto de los casos, se expresa a través del verbo: \#LaRiojaQueQuieres (Sánchez) o el pronombre \#ContigoSumamos (Casado).

Respecto a las funciones discursivas principales de estos hashtags, generalmente identifican en pocas palabras el tema del tuit, promoviendo la adhesión por parte de los usuarios -seguidores o no- al contenido del mensaje, lo que suele llevarse a cabo mediante el retuit, o bien insertando la etiqueta en el propio mensaje, de manera que se amplía la "conversación" en torno a ese tema. Se trata de cuestiones sobre las que el político quiere llamar la atención:

(12) @sanchezcastejon: Encuentro con organizaciones del ámbito de la \#digitalización y la \#ciencia. Junto a ellas queremos construir un programa para un modelo productivo más competitivo, una sociedad más preparada para afrontar el \#futuro, un país con menores desigualdades sociales. \#ConstruirGobierno (01-08-2019).

(13) @Santi_ABASCAL: ¿Nos quieren convencer de que SM el Rey tuvo que intervenir ante un problema de orden público, ante una algarada callejera? ¿Nos quieren convencer de que los golpistas no atacaron el orden 
Pano Alamán, A.

La política del hashtag en Twitter

constitucional? Nunca comulgaremos con ruedas de molino. \#Sentencia (14-102019).

Otras etiquetas tienen una función informativa. Por ejemplo, es frecuente que los topónimos se empleen para indicar el lugar en el que se celebrará el próximo mitin de un partido. Si bien, en otros casos, parecen tener una función contextualizadora cuando señalan encuentros internacionales o el lugar en el que han sido formuladas ciertas declaraciones, como señal demarcativa de una cita. En (14), por ejemplo, se citan en estilo directo, aun sin comillas, las palabras de Irene Montero, portavoz de Unidas Podemos, mientras participa en el primer debate televisado de la campaña del 10N, el "Debate a 7", emitido en TVE el 1 de noviembre de 2019:

(14) @PabloIglesias Estas propuestas para blindar los derechos laborales, hacer la vida más fácil a los autónomos y garantizar unas pensiones dignas a nuestros mayores son las que hacen que la CEOE no nos quiera en el Gobierno. @Irene_Montero_en \#Debatea7RTVE (01-11-2019).

Por último, destacan las etiquetas que constituyen actos de habla directivos con los que se incita a la participación. Algunas contienen el imperativo del verbo votar, que aparece fusionado con las siglas del partido para el que se solicita el apoyo. Se trata de consignas como \#VotaPSOE (Sánchez), \#VotaPP (Casado), \#VotaUnidasPodemos (Iglesias) y \#VotaVox, más tradicionales. Aparecen, aunque con poca frecuencia, en todos los perfiles, a excepción del de Rivera, que prefiere \#VamosCiudadanos. Con función similar se señalan \#VotaConElCorazón (Sánchez), \#VamosEspaña (Casado, Rivera), \#VotaFeminista (Iglesias) y \#PorEspañaVotaVox (Abascal). Como vemos, por un lado, emplean la consigna clásica con la que se invita explícitamente a votar al partido; por otro, adoptan fórmulas que, si bien no son originales puesto que se repiten o recuerdan lemas que han sido empleados en otros comicios (Garrido Lora, 2013), recurren, de forma diversificada, a expresiones propias del mundo deportivo ("vamos"), apelan a las emociones del elector ("con el corazón"), indican el motivo por el que cabe votarles ("por España) o bien señalan la causa o los valores que se defienden al votarles ("feminista"). En este sentido, asistimos a lo que Dader (2008: 234) llama "sensiocracia", una suerte de ideología del sentimiento en base a la cual procesamos las informaciones del entorno en términos de "empatía o dispatía sentimental" (Peña Jiménez y Ortiz Sobrino, 2011, p. 554). Es necesario precisar que la consigna, que se presenta como una "orden que una persona $u$ organismo dirigente da a los subordinados o afiliados" (DLE, 2014) en las agrupaciones políticas se distingue del eslogan o del lema de campaña, que parece tener un carácter más general, ya que expresa un objetivo o una promesa destinada a todos los electores.

\subsubsection{Etiquetas "electorales"}


Nos centrarnos ahora en el análisis de aquellas etiquetas que tienen vinculación con los lemas de las campañas electorales del 28A y 10N³. Como apuntábamos, se trata de las etiquetas que presentan mayor frecuencia en todos los casos, menos en el de Iglesias, en cuyos mensajes aparece una sola ocurrencia de la etiqueta-lema de la primera convocatoria electoral.

Tabla 2. Etiquetas que vehiculan el eslogan oficial de campaña.

\begin{tabular}{|l|l|l|l|l|l|}
\hline & SANCHEZ & CASADO & RIVERA & IGLESIAS & ABASCAL \\
\hline 28A & $\begin{array}{l}\text { \#HazQuePase } \\
(81)\end{array}$ & \#ValorSeguro (32) & $\begin{array}{l}\text { \#Vamos } \\
\text { Ciudadanos } \\
(12)\end{array}$ & $\begin{array}{l}\text { \#LaHistoriaLaE } \\
\text { scribesTú }\end{array}$ & \#PorEspaña (28) \\
\hline 10N & \#AhoraSí (58) & $\begin{array}{l}\text { \#PorTodoLoQue } \\
\text { NosUne (7) }\end{array}$ & $\begin{array}{l}\text { \#EspañaEnM } \\
\text { archa (17) }\end{array}$ & $\begin{array}{l}\text { \#UnGobiernoCo } \\
\text { ntigo (9) }\end{array}$ & $\begin{array}{l}\text { \#España } \\
\text { Siempre (60) }\end{array}$ \\
\hline
\end{tabular}

Cabe decir que \#AhoraSí y \#PorTodoLoQueNosUne llegaron a ser Trending Topic (TT) en España durante unas dos horas el primer día de campaña del 10N. Los datos que ofrece Trendinalia.com de los TT relativos al primero y al último día de campaña de las dos convocatorias consideradas indican que estos fueron los únicos hashtags electorales que llegaron a ser tendencia del momento ese día. Junto a estos, llegaron a ser TT las consignas y lemas: \#VotaPSOE (3h15') y \#VotaPP (2h30'), el 12A; \#EstaHistoriaLaEscribesTú (4h36') y \#VamosEspaña (2h30'), el 26A; \#CsEnMarcha (13h40'), el 1N; \#VotaUnidasPodemos (4h45') y \#VotaConEl Corazón (4h10'), el 8N.

Como el eslogan, estas etiquetas se caracterizan por la brevedad, la concisión, la simplicidad sintáctica y la densidad semántica. Se nota el recurso a fórmulas breves, elípticas incluso, que se apoyan en sintagmas escuetos, con dos o tres elementos fusionados con la inicial en mayúscula, algunos de los cuales son verbos. En los casos de Rivera y Abascal se adoptan fórmulas más breves, mientras que en los de Sánchez y Casado se alternan estructuras con dos y tres elementos según la campaña. Las etiquetas-eslogan de UnidasPodemos detectadas en los tuits de Iglesias presentan tres o más lexemas. En el caso de la convocatoria del 28A, se trata de un enunciado que pone el foco en "la historia" y en "tú" y que pretende subrayar la importancia del voto del ciudadano al que tutea, para decidir sobre el futuro del país, para escribir su "historia". En general, no se trata de fórmulas demasiado creativas, en particular, las que incluyen "España" (Rivera y Abascal), muy presente en las etiquetas y lemas de precampaña de otros partidos, como \#AhoraEspaña en los tuits de Sánchez (48 ocurrencias) y \#NosUneEspaña en los de Casado (9).

En cuanto al contenido, que aparece concentrado en pocas palabras, destacan los valores positivos: en el caso de Sánchez de forma explícita (\#AhoraSí); en otros casos derivan del significado positivo de ciertas palabras como, por ejemplo, el sustantivo "valor" y el adjetivo "seguro" en el primer lema de Casado, y la expresión "vamos" y

\footnotetext{
3 Sobre los lemas y estrategias comunicativas adoptadas por cada partido en ambas convocatorias, v. M. Á. Alfonso. Del 28A al 10N en los lemas de campaña. El Correo, 30-10-2019. Recuperado de: https://www.elcorreo.com/elecciones/generales/lemas-campana-partidos-20191030114715-ntrc.html
} 
la locución "en marcha", que presuponen movimiento y dinamismo, en los dos de Rivera. Más allá de las que se vinculan con los eslóganes, la mayor parte de las etiquetas analizadas en este estudio vehiculan mensajes que promueven la imagen positiva del líder-candidato y del propio partido. Sin embargo, en el caso de Rivera son frecuentes también hashtags como \#NoNosCallarán o \#ContraLaDespoblación, donde se destacan los elementos de polaridad negativa, una estrategia arriesgada que no sigue el resto de políticos. Por otra parte, los dispositivos más empleados durante la campaña buscan adular al votante. Se dirigen al elector como único capaz de hacer que "algo pase", en Sánchez. En otros casos, insertan la segunda persona del singular explícitamente para llamar a votar o para estar en el gobierno con ese partido, como en los hashtags de Iglesias; en otras aún se incluye un pronombre de primera persona plural inclusivo como "nos" para aludir de forma implícita a los valores e ideas que se comparten con el potencial elector. Se trata de informaciones que el destinatario del mensaje en Twitter debe inferir del contexto en que se inserta la etiqueta y de los conocimientos que comparte con el político. Por ejemplo, en (15), \#PorTodoLoQueNosUne deja espacio a todo tipo de interpretaciones sobre qué une al partido de Casado y al destinatario de su mensaje electoral. El cotexto del hashtag en ese tuit, en particular, la serie enumerativa "crear empleo, mejorar las pensiones, el campo...", da pistas al destinatario del líder del PP -principalmente, sus seguidores en la red-, sobre los temas que parecen preocupar y unir a ambos y los objetivos "por" los que habría que votarles. En (16), la colocación en secuencia de \#Oviedo y el lema \#PorEspaña invita a inferir que esa ciudad, o los residentes de esa ciudad que asisten al acto que se promueve, "está ahí" junto al líder y al partido, "por" el país:

(15) @pablocasado_: [...] Salimos a ganar las elecciones para crear empleo, mejorar las pensiones, el campo... \#PorTodoLoQueNosUne se abre una oportunidad de cambio ante este Gobierno que sufre España (08-10-2019).

(16) @Santi_ABASCAL: Falta más de una hora para el inicio del acto de VOX en Oviedo. Muchísimas personas ya guardan una larguísima cola para acceder al auditorio más grande de Oviedo. La \#EspañaViva esperanzada con VOX para el próximo 28-A. \#Oviedo \#PorEspaña (12-04-2019).

Desde el punto de vista del empleo de figuras retóricas, es posible afirmar que estas etiquetas-eslogan son poco creativas. Se apoyan principalmente en la elipsis (\#AhoraSí, \#EspañaEnMarcha, \#UnGobiernoContigo) y en analogías con el deporte, mediante expresiones prototípicas de ese mundo (\#VamosCiudadanos), y con los seguros y la finanza, a través del sintagma empleado frecuentemente en esos ámbitos (\#ValorSeguro). En todo caso, los referentes de las etiquetas pueden resultar opacos o difícilmente comprensibles para quienes no conocen la política española. En efecto, como afirman Peña Jiménez y Ortiz Sobrino, el contexto histórico, político y social adquieren una gran relevancia en este tipo de mensajes, ya que, como en los ejemplos, "estas palabras no pueden separarse en ningún caso de su situación de uso, ni del momento político en que han sido pronunciadas o utilizadas" (2011, p. 555).

Por otra parte, no se distancian demasiado de las que estos partidos han utilizado en citas electorales previas. En 2008, el PSOE adoptó el lema "Por todo lo que merece 
la pena" mientras que Unión Progreso y Democracia lanzó el titular "Lo que nos une" (Garrido Lora, 2013, p. 184). Se trata de dos lemas casi idénticos a "Por todo lo que nos une", empleado por el PP en la campaña del 10N. También en 2008, el PP apostó por "Con cabeza y corazón", que aunaba valores racionales y emocionales (Garrido Lora, 2013, p. 183). Con una estrategia similar se emplea en los tuits de Sánchez una de sus etiquetas-lema más frecuentes, "VotaConElCorazón", lanzada en ocasión de los comicios del $10 \mathrm{~N}$. Y es que, en los más de diez años que separan las elecciones de 2008 y de 2019, asistimos a la tendencia señalada por Peña Jiménez y Ortiz Sobrino (2011, p. 565), esto es, el "parecido in crescendo entre los eslóganes de los distintos partidos" y el empleo de:

lemas abiertos, poco concretos, sin rasgo ideológicamente marcados, de tal forma que no comprometen a sus promotores. Las campañas actuales persiguen por encima de todo ganar y no comprometerse. Plantean mensajes que puedan ser fácilmente compartidos por la mayoría (Peña Jiménez y Ortiz Sobrino, 2011, p. 560).

En lo que respecta a la perdurabilidad, rasgo característico del eslogan, Garrido Lora observa que "hoy se prefieren eslóganes más coyunturales" (2013, p. 179). Es este un aspecto que el empleo masivo de las redes sociales como Twitter por parte de los equipos de campaña ha acentuado cada vez más, a través de la condensación y fragmentación del mensaje político, en cierta medida incapaz de generar debate en la sociedad y trabajar con una perspectiva a largo plazo (Pano Alamán, 2019: 89).

\section{CONCLUSIONES}

El análisis llevado a cabo nos permite dar respuesta a las cuestiones planteadas al inicio de este estudio. En primer lugar, es posible afirmar que los hashtags son muy frecuentes en todos los perfiles analizados, en particular, en los del líder socialista, que emplea más de uno en la mayor parte de mensajes publicados entre el 12 de abril y el 8 de noviembre de 2019. Se trata, sin embargo, de etiquetas que se repiten en todos los perfiles, excepto en el de Iglesias, que hace un uso más diversificado de este dispositivo. Las etiquetas más frecuentes coinciden con los lemas y consignas de las campañas electorales, un dato que lleva a pensar que los políticos privilegian este empleo persuasivo de la etiqueta quizá por su forma breve y por su capacidad de generar "conversación" en ese contexto. No obstante, las estructuras son fácilmente intercambiables, puesto que se apoyan en significados polisémicos y abiertos para evitar, entre otras cosas, comprometerse.

Los hashtags analizados establecen asimismo relaciones internas con los elementos lingüísticos que aparecen en los tuits, y externas, cuando hacen referencia al contexto sociopolítico nacional e internacional. Estas se convierten no solo en mecanismos para contribuir a la difusión del propio argumentario político, sino que se convierten también en marcas de la enunciación dentro de actos asertivos, que indican cuál es su posición ante esos problemas. Con pocas diferencias entre los cinco líderes, muchas de las etiquetas empleadas suelen apoyarse en sustantivos aislados o en sintagmas nominales y verbales que tematizan el contenido del tuit, haciendo referencia a las 
Pano Alamán, A.

La política del hashtag en Twitter

cuestiones que preocupan al partido o que el líder pretende colocar en la agenda mediática y política, y a hechos de actualidad que denuncia o sobre los que toma una posición. Otras, generalmente topónimos, informan sobre el lugar de un evento, se insertan en expresiones de celebración, o indican el contexto en el que deben situarse unas declaraciones, en particular, en programas de televisión. En este caso, tienen funciones contextualizadoras y de demarcación de cita, que apelan especialmente a los conocimientos compartidos por el enunciador y el enunciatario.

Los hashtags aprovechan la brevedad y densidad semántica propias de los lemas y mensajes electorales para vehicular, en general, contenidos poco comprometidos y más afectivos que racionales, con el objetivo de viralizar sus mensajes y marcar los temas sobre los que se debate en Twiter. Este análisis ha mostrado que estos dispositivos replican las palabras y expresiones que ya se lanzan estratégicamente en otros medios y, puesto que parecen ignorar las etiquetas que emplean otros usuarios en Twitter, es posible afirmar que el empleo de la etiqueta por parte de los políticos es aún poco innovador. Una estrategia distinta pasaría por insertar en sus propios mensajes hashtags afines a sus ideas, empleados por sus seguidores, para participar en la "conversación" global.

\section{REFERENCIAS}

Briceño Romero, Y.; Calderón-Benavides, L.; Manrique, J. A. y Gélvez Salazar, C. (2019). Twitter en tiempos electorales. Una revisión de \#ColombiaDecide, en Anuario Electrónico de Estudios en Comunicación Social, 12(2), pp. 216-236.

Calvo Rubio, L. M. (2018). Twitter como segunda pantalla en los debates políticos en televisión. Análisis de la etiqueta \#L6Nrajoyrivera, en Icono 14, 16(1), pp. 160-184.

Escuder, S. (2019). Política en las redes, redes en la política: estudio de caso de la campaña \#VivirSinMiedo, en Teknokultura. Revista de Cultura Digital y Movimientos Sociales, 16(1), pp. 55-74.

Gallardo Paúls, B. y Enguix Oliver, S. (2016). Pseudopolítica: el discurso político de las redes sociales. Valencia: Universitat de València.

Garrido Lora, M. (2013). Estudio comparativo de los eslóganes electorales y comerciales: el caso de las elecciones generales españolas de 2008, en Convergencia, 20, pp. 173-192.

González Bengoechea, A. (2015). Redes sociales y política: El uso comunicativo de Twitter por parte de los principales partidos políticos, en Fòrum de recerca, 20, pp. 539-555.

Heyd, Th. y Puschmann, C. (2017). Hashtagging and functional shift: Adaptation and appropriation of the \#, en Journal of Pragmatics, 116, pp. 51-63. 
Pano Alamán, A.

La política del hashtag en Twitter

Held, G. (2011). Il testo breve: condensazione multimodale nella comunicazione di massa. En Held, G. y Schwarze, S. (Eds.), Testi brevi: teoria e pratica della testualità nell'era multimediale (pp. 15-30). Frankfurt am Main: Peter Lang.

López Eire, A. (1998). La Retórica en la publicidad. Madrid: Arco Libros.

Mancera Rueda, A. y Helfrich, U. (2014). La crisis en 140 caracteres: el discurso propagandístico en la red social Twitter, en Cultura, Lenguaje y Representación, Vol. XII, pp. 59-86.

Mancera Rueda, A. y Pano Alamán, A. (2013). El discurso político en Twitter. Barcelona: Anthropos.

Mancera Rueda, A. y Pano Alamán, A. (2015). Valores sintáctico-discursivos de las etiquetas en Twitter, en Círculo De Lingüística Aplicada a La Comunicación, 64, pp. 58-83.

Menna, L. (2012). Nuevas formas de significación en red: el uso de las \#etiquetas en el movimiento 15M, en Estudios de Lingüística del Español, 34. Recuperado de: http:/ / elies.rediris.es/elies34/Tesina_L-Menna.pdf. Última consulta: 24-07-2014.

Ortega, F. (2005). La fragmentación de la legitimidad política, en Revista Mexicana de Ciencias Políticas y Sociales, 47, Vol. 194, pp. 13-32. Recuperado de: http://revistas.unam.mx/index.php/rmcpys/article/view/42483/38599

Page, R. (2012). The linguistics of self-branding and micro-celebrity in Twitter: the role of hashtags, en Discourse Commun, 6, pp. 181-201.

Pano Alamán, A. (2019). Condensación y fragmentación del discurso político en Twitter. En Pellisser Rosell, N. y J. Oleaque Moreno (eds.) (2019). Mutaciones discursivas en el siglo XXI: la politica en los medios y las redes (pp. 75-92). València: Tirant lo Blanch.

Pano Alamán, A. y Mancera Rueda, A. (2014). La 'conversación' en Twitter: las unidades discursivas y el uso de marcadores interactivos en los intercambios con parlamentarios españoles en la red social, en Estudios de Lingüística del español, 35.1, pp. 234-268. Recuperado de: http://infoling.org/elies/35/elies35.1-10.pdf

Pellisser Rosell, N. y Oleaque Moreno, J. M. (Eds.) (2019). Mutaciones discursivas en el siglo XXI: la politica en los medios y las redes. València: Tirant lo Blanch.

Peña Jiménez, P. y Ortiz Sobrino, M. (2011). El eslogan político español en la campaña de elecciones generales de 2008, en Estudios sobre el Mensaje Periodístico, 17(2), pp. 549-568. 
Pano Alamán, A.

La política del hashtag en Twitter

Pérez Fumero, E. (2013). La enfermedad de Hugo Chávez en la agenda de medios públicos. Análisis del hashtag \#elmundoestaconchavez* desde la teoría del framing, en index comunicación, n. 3, pp. 63-85.

Reboul, O. (1978). El poder del eslogan. Valencia: Fernando Torres.

Santoveña, S. (2015). CiberSociedad y debate público en 140 caracteres: \#paro y \#corrupción, en Athenea Digital, 15(3), pp. 47-84. Recuperado de:

Scott, K. (2015). The pragmatics of hashtags: Inference and conversational style on Twitter, en Journal of Pragmatics, 81, pp. 8-20.

Zappavigna, M. (2011). Ambient affiliation: a linguistic perspective on Twitter, en New Media Society, 13(5), pp. 788-806.

Zeifer, B. (2020). El hashtag contestatario: cuando los hashtags tienen efectos políticos, en Dígitos. Revista de Comunicación Digital, 6, pp. 101-118.

\section{AUTOR/ES:}

\section{Ana Pano Alamán}

Ana Pano Alamán es Profesora Titular de Lengua y Lingüística Española en la Universidad de Bolonia. Sus principales áreas de investigación son el análisis lingüístico y pragmático del discurso digital y los discursos político y periodístico en español. Dentro de estas líneas ha publicado numerosos artículos en revistas nacionales e internacionales. Es autora de la monografía Dialogar en la Red (2008) y coautora de Political discourse on Twitter (2013), Colloquial Spanish on social networks (2013), Informatica umanistica (2017) y Opinar en la Red. Pragmatic analysis of the citizen voice (en prensa). Ha sido miembro del Consejo de Administración de la Asociación de Estudios sobre Discurso y Sociedad (EDiSo) y colabora con grupos de investigación y proyectos sobre el lenguaje en los medios, como Language and Press y METAPRES. The metalinguistic discourse in the Spanish press (1940-today).

ana.pano@unibo.it

Orcid ID: https:/ / orcid.org/0000-0003-3652-8694

Google Scholar: https:// scholar.google.com/citations?user=nfz8MBEAAAAJ\&hl=es

ResearchGate: https://www.researchgate.net/profile/Ana_Pano_Alaman

Scopus: $\underline{56023208800}$

Academia.edu: https:// unibo.academia.edu/AnaPanoAlamán 